\title{
Sleep and Circadian Rhythm in Critical Illness
}

Irene Telias ${ }^{1,2,3}$ and Mary Elizabeth Wilcox ${ }^{1,4^{*}}$

\begin{abstract}
This article is one of ten reviews selected from the Annual Update in Intensive Care and Emergency Medicine 2019. Other selected articles can be found online at https://www.biomedcentral.com/collections/ annualupdate2019. Further information about the Annual Update in Intensive Care and Emergency Medicine is available from http://www.springer.com/ series/8901.
\end{abstract}

\section{Introduction}

Sleep is controlled by two major regulatory systems: a circadian system that drives 24-h periodicity (Process C), and a homeostatic system (Process S) that ensures adequate amounts of sleep are obtained. Both processes are disturbed in critically ill patients, potentially due to exposure to sleep-altering medications (e.g., propofol), the structure of the intensive care unit (ICU) environment (e.g., workflow), aggravation of a pre-existing sleep disorder, and/or effects of acute illness (e.g., sepsis). As a result, patients may experience delirium, poor respiratory function, and dysregulated immune system reactivity. Several methods of measuring sleep in the ICU exist, although all provide their own challenges. A number of intervention-based therapies to improve ICU sleep and circadian rhythm disturbances have been explored, including noise reduction protocols, music therapy, light treatment, and different modes of mechanical ventilation. These studies have met with limited success.

\section{Physiology of sleep}

The brain is active during sleep. Sleep is regulated by several centers in the brainstem, hypothalamus, thalamus and forebrain. Involvement and downregulation of the

\footnotetext{
*Correspondence: elizabeth.wilcox@utoronto.ca

${ }^{1}$ Interdepartmental Division of Critical Care Medicine, University of Toronto, Toronto, Canada

${ }^{4}$ Division of Respirology, Department of Medicine, Toronto Western Hospital, Toronto, ON, Canada

Full list of author information is available at the end of the article
}

ascending reticular activating system (ARAS) is important for sleep-wake regulation and involves a number of nuclei in the hypocretinergic, GABAergic, histaminergic, adrenergic, and cholinergic systems. Together, these systems orchestrate sleep into the main phases of rapid eye movement (REM) and non-REM (NREM) sleep, classically described by the Rechtschaffen and Kales Rules (R\&K rules) [1]. NREM sleep is further divided into three sleep stages: substages N1, N2 and N3. The N3 substage is known as slow wave sleep (SWS) [1]. Normal sleep architecture consists of stages occurring in cycles of 90-120 min each [1, 2].

Circadian rhythms refer to self-sustained fluctuations with a period of approximately (cira) 1 day (diem) in various physiological processes. In humans, the circadian system is composed of many individual, tissue-specific clocks with their phase being controlled by the master circadian pacemaker, the suprachiasmatic nucleus $(\mathrm{SCN})$ of the hypothalamus [2]. The most evident circadian rhythm in humans is the sleep-wake cycle. The SCN directly regulates multiple neurotransmitter systems that either drive or modulate sleep, including the hypothalamo-pituitary-adrenal (HPA) axis and melatonin from the pineal gland [2]. Circadian clock genes identified in human peripheral tissues to date include Period (Per-1-3), Cryptochrome (Cry-1 and Cry-2), Clock, and Bmal1, which coordinate with the master circadian pacer [2]. External factors that are called 'timekeepers' or zeitgebers, such as light/dark cycle, interact with internal clocks by synchronizing their different oscillation phases. Circadian rhythms have a duration of approximately $24 \mathrm{~h}$ and can be assessed through chronobiologic analysis of the time series of melatonin, cortisol and temperature [2].

\section{Altered sleep in the ICU}

Normal sleep architecture varies among individuals [3]. A 'normal' sleep stage in a healthy young adult might be: $2-5 \% \mathrm{~N} 1,45-55 \% \mathrm{~N} 2,3-15 \% \mathrm{~N} 3$ or SWS and $20-25 \%$ REM [3]. Normal transition from wake to sleep onset occurs within 10-20 min, and the first period of REM typically occurs within 90-120 min [2]. Although the total sleep time within a $24-\mathrm{h}$ period in the ICU is 
similar to that of a non-hospitalized individual, marked differences exist in sleep architecture. As much as half of a critically ill patient's sleep occurs during daytime hours with N1 and N2 representing a larger percentage of the total sleep time. The duration and frequency of both SWS and REM sleep are reduced, and frequent arousals lead to high sleep fragmentation. Altered patterns of sleep during an ICU stay take days to normalize and in certain cases may persist after transfer to the general floor/ward. In a recent study by Wilcox et al. characterizing the quality and quantity of sleep in ICU survivors within 7 days of discharge to the ward, approximately two-thirds $(61 \%)$ of patients had persistent sleep disturbances. Further, patients continued to experience little or no SWS and/or REM sleep, independent of external factors (e.g., frequency of vital sign measurement or number of beds in the room) [4].

Critically ill patients experience circadian rhythm disruption likely secondary to the absence of effective zeitgebers in the ICU environment. In addition, systemic inflammation may also disrupt circadian rhythmicity of chronobiologic markers. In a study by Haimovich et al., the administration of intravenous endotoxin in human volunteers dramatically altered circadian clock gene expression in peripheral blood leukocytes, suggesting a misalignment of central and peripheral clocks in the modulation of the inflammatory response [5]. Mundigler et al. assessed circadian disruption in 17 septic patients, 7 non-septic patients and 21 controls admitted to an ICU [6]. Urinary 6-sulfatoxymelatonin (6-SMT) exhibited loss of circadian rhythmicity with no daytime decline in septic patients [6]. Recently, Li et al. measured plasma levels of melatonin, tumor necrosis factor (TNF)- $\alpha$, interleukin (IL)- 6 and messenger RNA of the circadian genes Cry-1 and Per-2 for 24-h in septic and non-septic ICU patients $(n=22)$ [7]. Altered circadian rhythm of melatonin secretion, reduced expression of Cry-1 and Per-2, and elevated levels of TNF- $\alpha$ and IL- 6 were seen in patients with sepsis [7]. Further, peripheral circadian gene expression was suppressed independent of the melatonin rhythmicity, confirming that, at least in the acute phase of sepsis, there is an uncoupling of the central master clock and peripheral tissue-specific clock genes.

\section{Mechanisms and physiological consequences of sleep disturbances in the ICU Light-dark cycle}

Light is measured in units of lux. On a sunny day in early spring, light levels range from 32,000 to 60,000 lux. In the ICU, reported daytime light levels range from mean illumination levels of 30-165 lux; nocturnal light levels vary from 2.4 to 145 lux; and during procedures (e.g., central line insertion) light devices can deliver up to 10,000 lux $[8,9]$, which definitely can alter a patient's circadian rhythm. Different studies modulating light exposure, have been shown to decrease incident delirium, possibly through a mechanistic link with modulation of sleep. For example, nocturnal light exposure decreases the secretion of melatonin, a hormone secreted by the pineal gland in response to darkness, which can ultimately result in sleep disruption [2]; the disruption of the circadian rhythm seen in patients with severe sepsis $(n=7)$ was reflected in the first $48 \mathrm{~h}$ of ICU admission by disordered diurnal variation of urinary 6-SMT excretion [10]. In animal models, circadian rhythm disruption due to constant light exposure led to reduced expression of Per-2 in the $\mathrm{SCN}$ and subsequent behavioral symptoms of delirium (i.e., executive dysfunction and memory impairment) [11]. Clinical symptoms were reversed with nobiletin, a known enhancer of Per-2 function [11]. Findings of diurnal disruption have been reported in traumatic brain injury (TBI), trauma and medical patient populations [12]; any association between diurnal disruption and resultant outcome (e.g., sedative use, incidence delirium or length of stay) in these patient populations has yet to be demonstrated.

\section{Noise in the ICU}

Noise has been reported as an important contributing factor to sleep disturbance in the ICU. The most common contributing sources of sound disruption are staff conversation, alarms, and patient care interventions [13]. As per the World Health Organization, sound levels should not exceed 30 A-weighted decibels (dBA). Sound levels in numerous ICU studies have reported levels of noise at mean values of 53-59 dBA with peak noise levels of 67-86 dBA $[13,14]$. ICU noise levels during daytime and nighttime hours have been found to be similar. ICU noise contributes to patients' lack of REM sleep [13].

\section{Sensorimotor experience Sedation}

Unlike natural sleep that serves an essential biological function, sedation frequently leads to atypical electroencephalogram (EEG) patterns that are not commonly observed in normal sleep. Benzodiazepines and propofol, both GABA agonists, are frequently used for sedation in critically ill patients, propofol being recommended as a first-line agent by current guidelines [15]. Benzodiazepine administration results in decreased sleep latency but adversely effects sleep architecture, decreasing SWS and REM stages of sleep [16]. Propofol is also a potent suppressor of SWS and at high doses can induce EEG burst suppression. Opioids, commonly administered in conjunction with sedatives in critically ill patients, bind the $\mu$-receptors of the ponto-thalamic arousal pathway, a key pathway in REM generation [17]. In a dose-dependent manner, opioids can suppress both SWS and REM [17]. In 
an observational study $(n=21)$ of mechanically ventilated medical ICU patients on intravenous sedation and analgesia, pronounced temporal disorganization and a paucity of normal sleep EEG findings were seen [18]. Although circadian rhythm was preserved in this study, patients exhibited a phase delay in their excretion of urinary 6-SMT, suggesting that their circadian pacemakers were free-running [18].

Dexmedetomidine is one of the most recently introduced agents for sedation in the ICU. It is a potent and highly selective $\alpha$-2-adrenergic agonist, with the action of dose-dependent sedation, anti-anxiolysis, and analgesia adjunct. Dexmedetomidine has been shown to more closely create natural sleep than other GABA agonist agents. In two small pilot studies, one study showed improved sleep efficiency and sleep time at night with dexmedetomidine [19] and another study also demonstrated improved sleep efficiency and stage 2 sleep as well as a modification of sleep pattern, shifting sleep (i.e., more than $75 \%$ total sleep time) to evening hours [20]. Recently, low-dose nocturnal dexmedetomidine was found to reduce the incidence of delirium in the ICU, without having an effect on patient-reported sleep quality [21]. Numerous outstanding questions remain in investigating the interplay of sleep, circadian rhythm and sedatives in the ICU. Efforts to date have focused on minimizing administration of these agents whilst mechanistic investigations are ongoing.

\section{Restraint use}

Conditions encountered by a patient in the ICU could resemble those deliberately created for experiments of sensory and perceptual deprivation. The use of physical restraints deprives patients of a normal sensory interaction with their environment. Short-term arm immobilization was shown in healthy volunteers to reduce local synaptic activity in sensorimotor areas, suggesting that cortical plasticity may be linked to local sleep regulation [22]. A study exploring the impact of physical restraints on sleep quantity and quality would help in informing care recommendations.

\section{Organ function \\ Mechanical ventilation}

Sleep disturbances in patients under mechanical ventilation are key; however, the interaction between sleep and mechanical ventilation is complex. There is a pathophysiological link between patient-ventilator interaction and sleep disturbances directly or through a necessity for higher doses of sedative drugs. In addition, sleep disturbances in and of themselves, and incident delirium, possibly due to a need for more sedation may all lead to prolonged weaning course and lengthier durations of mechanical ventilation.
Over assistance during pressure-support ventilation (PSV) leads to sleep disruption. During sleep, ventilatory demand, respiratory drive and inspiratory effort decrease. Therefore, it is not uncommon for ventilatory support during PSV to become excessive in relatively normal lungs. This results in hyperventilation and $\mathrm{PaCO}_{2}$ decreasing below the apnea threshold. Asynchronies, related to a high respiratory drive, such as flow starvation, short cycling, and double triggering might theoretically contribute to sleep disturbances and delirium in the context of air hunger necessitating greater sedative exposure [23]. On the other hand, patient-ventilator asynchronies are associated with longer duration of mechanical ventilation that might be associated, at least in part with disturbances of sleep. Thille et al. recently demonstrated that the weaning process was longer in patients who, after failing the first spontaneous breathing trial (SBT), had atypical sleep and an absence of REM as compared to those exhibiting a normal sleep pattern [24]. Preliminary data from a physiological study assessing the relationship between quantity and quality of sleep and weaning outcome shows that patients who passed an SBT and were successfully extubated had a polysomnography (PSG) trace compatible with being more awake (assessed by the odds ratio product [25]) compared to those who failed an SBT or passed but were not extubated (Martin Dres, personal communication). This complex interaction might be mediated by higher use of sedatives, as described by Mehta et al. [26], where patients receiving higher doses of sedatives and opioids at night were more likely to fail readinessto-wean criteria, fail a SBT, or not be extubated despite having passed an SBT after clinical assessment. Again, in the preliminary data by Dres et al. (personal communication), patients who failed the SBT were shown to have had lesser degrees of interhemispheric correlation during sleep. This is consistent with a study of delirious patients $(n=70)$, where reductions in peak, mean, and total amplitude of urinary 6-SMT was associated with inability to wean [27]. Although circadian rhythm may influence one's ability to wean, how this effect is mediated through incident delirium or on the weaning process directly is unknown.

\section{Immune system}

Melatonin, in addition to mediating the effects of the photoperiod, also plays an important role in the adaptive response of an organism. Experimental studies have shown that binding of melatonin to specific receptors in antigen-activated Type $1 \mathrm{~T}$-helper cells (Th-1) upregulates pro-inflammatory cytokine production as well as enhancing phagocytosis and antigen presentation [28]. Animal models have demonstrated a protective effect of melatonin against lethal viral encephalitis, infectious hepatitis, and 
hemorrhagic or septic shock; it has been shown to inhibit TNF- $\alpha$, and to reduce post-shock levels of IL- 6 , superoxide production in the aorta, and inducible nitric oxide synthase (iNOS) in the liver preventing endotoxin induced circulatory shock [28]. Altered patterns of illumination in the ICU have been found to abolish the physiological regulation of melatonin secretion in response to darkness and light [29]; this pathway is directly linked to the inflammatory response and possibly mortality. For a complete review of circadian aspects of the immune response see Papaioanno et al. [28].

\section{Nutrition}

Although guidelines advise that enteral nutrition be initiated within $24-48 \mathrm{~h}$ in the critically ill patient [30] there is no specific recommendation around the timing of nutrition delivery (e.g., daytime hours versus continuous 24-h feeds). Peripheral circadian oscillators are sensitive to stimuli associated with food intake, enabling animals to uncouple rhythms of behavior and physiology from light-dark cycles and instead align them with predictable mealtimes. In our experience, the current practice in the ICU is to administer feeds, as tolerated, over a 24-h period with multiple interruptions during the day for procedures (e.g., bronchoscopy), medication administration (e.g., levothyroxine) or radiographic tests. It may be reasonable to consider the restriction of feeds to daytime hours to assist in the re-entrainment of the SCN. The literature is currently primed for a survey of ICU practices around the timing of feeds (e.g., daytime, 24-h) as this information may help to inform how to best study the impact of nutrition delivery on circadian rhythm disruption in the ICU.

\section{Delirium and other neuropsychological sequelae}

Studies have found a correlation between sleep deprivation and mental status changes in the ICU. Delirium is characterized by inattention, fluctuating mental status, disorganized thinking and an altered level of consciousness, findings which are also characteristics of sleep deprivation. Sleep disturbances are common in delirious patients. While sleep deprivation is regarded as a potentially modifiable risk factor for the development of delirium, it is also possible that delirium itself may contribute to experienced sleep disturbances. Studies conducted mainly in cardiac surgical patients indicate that sleep deprivation can cause [31], be a result of [32], or simply lower the threshold for transitioning to delirium. Decreased SWS and REM sleep have been hypothesized to contribute to developing delirium. A recent study of ICU patients demonstrated an association between delirium and severe REM sleep reduction $(<6 \%$ of total sleep time), however a causal relationship was not clearly established [33]. The association between sleep disturbance and delirium remains unclear, but it is possible that their relationship shares a common pathophysiologic pathway.
There is considerable evidence linking sleep-related breathing disorders and poor sleep quality with cognitive impairment in many patient populations. Cognitive domains particularly associated with sleep disruption include working memory, semantic memory, processing speed, and visuospatial abilities [34]. Experimental studies support a number of potential neurobiological mechanisms including accumulation of beta-amyloid pathology, abnormalities of tau, synaptic abnormalities, changes in hippocampal long-term potentiation, impaired hippocampal neurogenesis, and gene expression changes. Few studies have rigorously evaluated the prevalence of sleep disruption after critical illness and its potential role in potentiating cognitive impairment. A recent systematic review by Altman and colleagues reported on 22 studies examining sleep after hospital discharge in survivors of critical illness; however, none of these studies reported on cognitive outcomes [35]. Despite sleep disturbances improving over time, up to two-thirds (61\%) of patients reported persistently poor sleep at 6 months follow-up [35]. Analyses of risk factors for sleep disturbances have had conflicting results, but persistent sleep disturbances were consistently associated with post-discharge psychological comorbidities and impaired quality of life.

The risk of developing psychological morbidity after discharge from intensive care is as high as $60 \%$ [36]; psychological morbidity includes depression, anxiety and posttraumatic stress disorder. Numerous follow-up studies have demonstrated an association between depressive symptoms and increased levels of fatigue, stress, and anxiety in healthy participants subject to sleep restriction. The underlying mechanism between sleep, circadian rhythm disruption and depression is not well understood; it is theorized that generation of sleep and mental health disorders share overlapping neural mechanisms such that defects in these endogenous pathways result in pathologies to both behaviors. Sleep and circadian rhythm disruption after critical illness may contribute to post-ICU psychological disorders, such as depression; associated risks may bechallenging to understand given the complexities of pre-existing comorbid conditions and ICU exposures (e.g., sedation).

\section{Sleep measurement in the ICU}

Sleep can be measured using a variety of objective and subjective techniques. The gold standard for the objective measurement of sleep is laboratory-based PSG. PSG is a multi-parametric test that monitors brain activity by EEG, eye muscles (electrooculography), muscle activity or skeletal muscle activation (electromyography) and heart rhythm. It is the only method of sleep measurement capable of identifying individual sleep stages following the $\mathrm{R} \& \mathrm{~K}$ rules; these stages are scored epoch-by-epoch in 
accordance with the American Association of Sleep Medicine criteria [1]. The application of conventional classification criteria is challenging in the ICU as alteration in cerebral metabolism, electrolyte disorders, intoxications, and medications influence sleep patterns. Alternative or supplementary criteria for PSG scoring have been proposed by Drouot et al. separating EEG recordings into states of either pathological wakefulness or atypical sleep. Devised and validated on non-sedated patients in the ICU, this method of scoring predicted atypical sleep with a sensitivity of $100 \%$ and a specificity of $97 \%$ [37].

As classical R\&K sleep stages are discrete, they are unable to describe the continuum between wakefulness and sleep. Recently, an automated algorithm named odds ratio product was developed that enables continuous measurement of sleep state ranging from full wakefulness (2.5) to deep sleep (0) [25]. EEG is assessed by rating epochs based on the relative power spectrum of each frequency band (delta, theta, alpha-sigma and beta). In a validation dataset of outpatient PSG recordings, an odds ratio product $<1$ predicted sleep and odds ratio product $>2$ wakefulness, with 95\% accuracy. Additionally, correlation $\left(r^{2}=0.98\right)$ was high between the odds ratio product and the probability of arousals and awakenings. Formal validation in a cohort of ICU patients has yet to be performed.

Bispectral index (BIS), an EEG-derived method for assessing the depth of sedation, mainly used during general anesthesia in the operating room, has been proposed as an alternative means of sleep assessment. Unfortunately, BIS is sensitive to technique and its interpretation is difficult. Further, its use for sleep assessment is poorly documented. Spectral edge frequency has been evaluated to assess sleep states as well as circadian rhythmicity [18], but suffers from inconsistency in selecting which epochs to include. In addition, further studies are needed to determine its validity in an ICU population.

Actigraphy, which continuously measures an individual's movement using a wrist-watch-like device on the wrist or ankle, is another alternative to PSG. The presence of movement indicates wakefulness, and its absence indicates sleep. This widely-used method has been validated in several populations for measurement of total sleep time and sleep fragmentation [38]. Actigraphy has been validated against biochemical markers of circadian rhythmicity [39]. A recent systematic review of actigraphy in the ICU showed that when compared to PSG, nurse assessment and patient questionnaires, actigraphy tended to consistently overestimate total sleep time and sleep efficiency [40]. When compared to PSG, actigraphy under-reported nocturnal awakenings. Overall awakenings were more frequently reported by nurse assessment and patient questionnaires compared to actigraphic recordings in the ICU.
The use of subjective measures of sleep assessment, such as patient or nurse questionnaires, is simple, easy and relatively inexpensive compared to other objective measures of sleep. Patients may keep daily sleep diaries or a sleep log. The Richards-Campbell Sleep Questionnaire (RCSQ), the Sleep in the Intensive Care Unit Questionnaire, and the Verran/Snyder Halpern Sleep Scale have all been tested in ICU patient populations [41]. Incident delirium and the frequent use of sedatives limit the use of instruments. Further, they typically report only on nighttime sleep, whereas sleep in the ICU is distributed over a 24-h period. Nursing assessment using the Echols Sleep Behavior Observation Tool, Nurses' Observation Checklist and the RCSQ can be used to estimate sleep [41]. Nursing-derived assessments however tend to overestimate total sleep time and sleep efficiency but underestimate awakenings when compared to PSG. Subjective assessments of sleep are variably reliable and provide no information on experienced sleep stages or circadian rhythmicity, limiting their utility in assessing sleep outcomes the ICU.

\section{Efforts to improve sleep in the ICU}

A number of studies have evaluated interventions targeting sleep optimization in the ICU, including non-pharmacologic sleep bundles, bright light therapy, earplugs, pharmacologic therapy, relaxation techniques and differing modes of mechanical ventilation, with mixed results. While it is likely that a successful sleep improvement program will need to address both internal and external factors disturbing ICU sleep, no single study has strongly proven its efficacy as an intervention (Fig. 1). The widespread use of an ICU specific sleep protocol would require a substantial commitment on the part of the individual center for its implementation. Precipitating such a culture change would likely require the demonstration of a substantial outcome benefit justifying a change to long-held workflow and care provision habits.

\section{Reducing environmental impact on sleep}

As the duration, intensity, and wavelength of a light stimulus modulates circadian rhythm via the central circadian clock, it makes intuitive sense to try to restore temporal disorganization of circadian pacemakers by modulating light exposure in the ICU. A single center randomized interventional study $(n=11)$ evaluating two different light exposures in postoperative patients with esophageal cancer, showed that subjects provided with more intense light had lower incident delirium [42]. Further, in a multicenter, prospective study of 523 non-intubated patients, delirium incidence was reduced in those exposed to visible sunlight in their hospital room [43]. In contrast, however, a large study of medical ICU patients $(n=3577)$ exposed to differing ambient light 


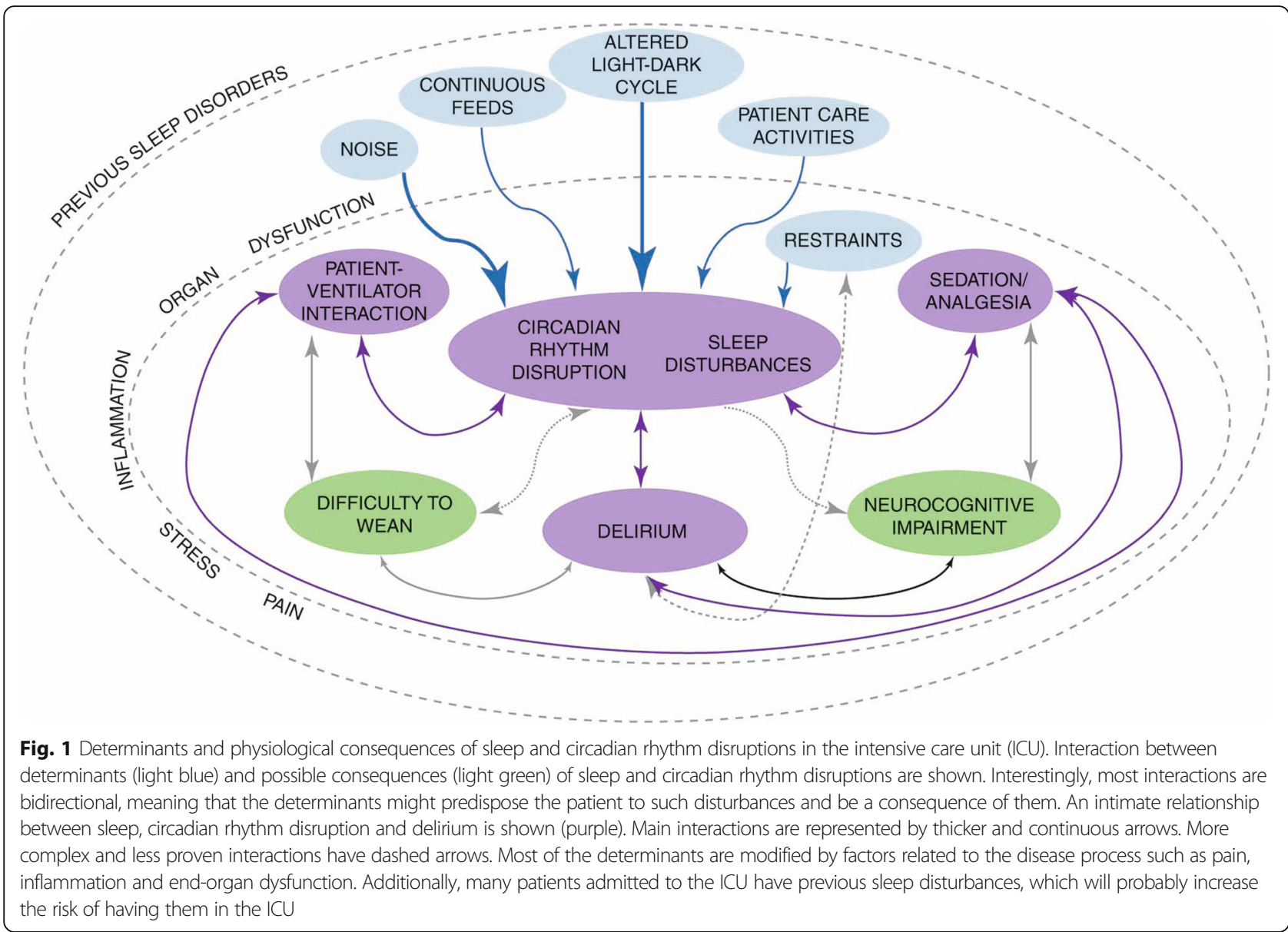

levels, with an approximately threefold difference in mean light levels due to different room orientations (south facing $399.2 \pm 146$ lux as compared to east facing rooms $30.6 \pm 1.3$ lux), there were no associated differences in sedative, analgesic or neuroleptic use, suggesting no impact on delirium incidence [9]. Conversely, in a small single center prospective study of elderly patients admitted for acute cardiac, respiratory or renal diseases ( $n$ $=10$ ), decreases in nocturnal lighting thresholds led to progressive resynchronization of circadian rhythm; circadian rhythms were severely altered during the first $24 \mathrm{~h}$ but progressively resynchronized by day 5 [44].

The effects of noise reduction strategies on sleep in the ICU remain controversial. Demoule et al. found that in non-sedated ICU patients, adding earplugs and eye masks to standard care reduced anxiety and improved sleep quality, specifically reducing long awakenings and increasing the duration of SWS [45]. Beneficial effects of noise reduction may be limited to the early stage of ICU admission [43]. Limitations of these studies include small sample sizes, lack of standard measures, and before-after study design. Well-designed trials of environmental interventions with adequate sample sizes are needed.

\section{Pharmacological considerations}

Pharmacological therapy for sleep and circadian rhythm disruption includes a careful review of existing pharmacological treatments, including the omission of chronic medications that may lead to withdrawal symptomatology. As many of the medications administered in the ICU have effects on normal sleep physiology, if medications such as opioids or sedatives cannot be discontinued then their administration should be limited to a minimal effective dose. Medications specifically used for acute sleep disturbances should be used for short periods with ongoing reassessment of necessity. Further, any medication prescribed for sleep should be accompanied by non-pharmacologic interventions (e.g., daytime mobilization and attempts at maintaining daytime wakefulness). Although medications for acute sleep disturbances may increase total sleep time they may not improve sleep quality. As an example, a small randomized controlled trial was able to show that patients who received $10 \mathrm{mg}$ of oral melatonin as compared to placebo demonstrated improved nocturnal sleep efficiency as measured using BIS [46]. Pharmacokinetic analyses suggested that $10 \mathrm{mg}$ dosing was too high, and that $1-2$ $\mathrm{mg}$ of melatonin could be used in future studies. Studies of melatonin, sleep and delirium are ongoing. 


\section{Mechanical ventilation}

Mechanical ventilation represents another important potential cause of sleep disruption. Reducing patientventilator asynchrony by optimizing ventilatory settings has been shown to mitigate the impact of mechanical ventilation on sleep [47]. Improved sleep efficiency has been achieved either by limiting the amount of ventilatory support $[48,49]$ or by altering ventilatory modes (e.g., proportional assist ventilation or neutrally-adjusted ventilatory assist modes). Surprisingly, few studies have examined the effect of time of day on minute ventilation. Recently, difficult to wean tracheostomized patients were reconnected to the ventilator at night to improve sleep efficiency [50]. Airway caliber is known to vary in healthy human beings across a 24-h period. In asthmatics, airflow obstruction tends to worsen in the early morning, corresponding to circadian changes in pulmonary function and abundance of immune cells in the airway. Little is known about how the molecular clock controls lung physiological function and how its disruption might influence respiratory mechanics during ventilation or weaning processes.

\section{Conclusion}

There are complex interactions between physiological, behavioral and environmental factors that contribute to sleep and circadian rhythm disturbances in ICU patients. Little is known about the exact mechanisms leading to these disruptions or the importance of each factor individually on the experienced sleep disturbance. The relationship between sleep, circadian rhythm and outcome after critical illness requires further study. Moreover, measurement of sleep quantity and quality is technically difficult, creating further challenges in delineating relationships. Efforts to date have been focused on non-pharmacologic and pharmacological strategies to improve sleep, with some promising results; however, for broader implementation a change in culture needs to occur. Less attention has been directed towards understanding underlying biological mechanisms of disturbed sleep and circadian disruption. Designing interventions grounded in basic biological principles might prove more effective in improving sleep in the ICU. Without strong evidence of benefit to facilitate changes in practice, the implementation of sleep-specific improvement bundles will be challenging.

\section{Acknowledgements}

None.

Funding

This study received no funding. Publication costs were funded by the authors.

Availability of data and materials

Not applicable.
Authors' contributions

IT and MEW conceived the idea for the manuscript. MEW wrote the initial draft. Both authors read and approved the final manuscript.

Ethics approval and consent to participate

Not applicable.

Consent for publication

Not applicable.

Competing interests

The authors declare that they have no competing interests.

\section{Publisher's Note}

Springer Nature remains neutral with regard to jurisdictional claims in published maps and institutional affiliations.

\section{Author details}

${ }^{1}$ Interdepartmental Division of Critical Care Medicine, University of Toronto, Toronto, Canada. ${ }^{2}$ Keenan Research Centre, Li Ka Shing Knowledge Institute, St. Michael's Hospital, Toronto, Canada. ${ }^{3} \mathrm{Critical}$ Care Medicine, University Health Network and Sinai Health System, Toronto, Canada. ${ }^{4}$ Division of Respirology, Department of Medicine, Toronto Western Hospital, Toronto, ON, Canada.

Published online: 09 March 2019

References

1. American Academy of Sleep Medicine. The AASM manual for the scoring of sleep and associated events: rules, terminology and technical specifications, version 2.5. Westchester: American Academy of Sleep Medicine; 2018.

2. Collop NA, Salas RE, Delayo M, Gamaldo C. Normal sleep and circadian processes. Crit Care Clin. 2008;24:449-60.

3. Hirshkowitz M, Whiton K, Albert SM, et al. National Sleep Foundation's sleep time duration recommendations: methodology and results summary. Sleep Health. 2015;1:40-3.

4. Wilcox ME, Lim AS, Pinto R, Black SE, McAndrews MP, Rubenfeld GD. Sleep on the ward in intensive care unit survivors: a case series of polysomnography. Intern Med J. 2018;48:795-802.

5. Haimovich B, Calvano J, Haimovich AD, Calvano SE, Coyle SM, Lowry SF. In vivo endotoxin synchronizes and suppresses clock gene expression in human peripheral blood leukocytes. Crit Care Med. 2010;38:751-8.

6. Mundigler G, Delle-Karth $\mathrm{G}$, Koreny M, et al. Impaired circadian rhythm of melatonin secretion in sedated critically ill patients with severe sepsis. Crit Care Med. 2002:30:536-40.

7. Li CX, Liang DD, Xie GH, et al. Altered melatonin secretion and circadian gene expression with increased proinflammatory cytokine expression in early-stage sepsis patients. Mol Med Rep. 2013;7:1117-22.

8. Dennis CM, Lee R, Woodard EK, Szalaj JJ, Walker CA. Benefits of quiet time for neuro-intensive care patients. J Neurosci Nurs. 2010;42:217-24.

9. Verceles AC, Liu X, Terrin ML, et al. Ambient light levels and critical care outcomes. J Crit Care. 2013;28:110 e111-8.

10. Verceles AC, Silhan L, Terrin M, Netzer G, Shanholtz C, Scharf SM. Circadian rhythm disruption in severe sepsis: the effect of ambient light on urinary 6sulfatoxymelatonin secretion. Intensive Care Med. 2012;38:804-10.

11. Gile J, Scott B, Eckle T. The period 2 enhancer nobiletin as novel therapy in murine models of circadian disruption resembling delirium. Crit Care Med. 2018:46:e600-8.

12. Seifman MA, Gomes K, Nguyen PN, et al. Measurement of serum melatonin in intensive care unit patients: changes in traumatic brain injury, trauma, and medical conditions. Front Neurol. 2014;5:237.

13. Freedman NS, Gazendam J, Levan L, Pack Al, Schwab RJ. Abnormal sleep/ wake cycles and the effect of environmental noise on sleep disruption in the intensive care unit. Am J Respir Crit Care Med. 2001;163:451-7.

14. Freedman NS, Kotzer N, Schwab RJ. Patient perception of sleep quality and etiology of sleep disruption in the intensive care unit. Am J Respir Crit Care Med. 1999:159:1155-62.

15. Barr J, Fraser GL, Puntillo K, et al. Clinical practice guidelines for the management of pain, agitation, and delirium in adult patients in the intensive care unit. Crit Care Med. 2013;41:263-306. 
16. Plante DT, Goldstein MR, Cook JD, et al. Effects of oral temazepam on sleep spindles during non-rapid eye movement sleep: a high-density EEG investigation. Eur Neuropsychopharmacol. 2015;25:1600-10.

17. Dimsdale JE, Norman D, DeJardin D, Wallace MS. The effect of opioids on sleep architecture. J Clin Sleep Med. 2007;3:33-6.

18. Gehlbach BK, Chapotot F, Leproult R, et al. Temporal disorganization of circadian rhythmicity and sleep-wake regulation in mechanically ventilated patients receiving continuous intravenous sedation. Sleep. 2012;35:1105-14.

19. Lu W, Fu Q, Luo X, Fu S, Hu K. Effects of dexmedetomidine on sleep quality of patients after surgery without mechanical ventilation in ICU. Medicine (Baltimore). 2017;96:e7081.

20. Alexopoulou C, Kondili E, Diamantaki E, et al. Effects of dexmedetomidine on sleep quality in critically ill patients: a pilot study. Anesthesiology. 2014 121:801-7.

21. Skrobik Y, Duprey MS, Hill NS, Devlin JW. Low-dose nocturnal dexmedetomidine prevents icu delirium. A randomized, placebo-controlled trial. Am J Respir Crit Care Med. 2018;197:1147-56.

22. Huber R, Ghilardi MF, Massimini M, et al. Arm immobilization causes cortical plastic changes and locally decreases sleep slow wave activity. Nat Neurosci. 2006;9:1169-76

23. Pham T, Telias I, Piraino T, Yoshida T, Brochard LJ. Asynchrony consequences and management. Crit Care Clin. 2018;34:325-41.

24. Thille AW, Reynaud F, Marie D, et al. Impact of sleep alterations on weaning duration in mechanically ventilated patients: a prospective study. Eur Respir J. 2018:51:1702465.

25. Younes M, Ostrowski M, Soiferman M, et al. Odds ratio product of sleep EEG as a continuous measure of sleep state. Sleep. 2015;38:641-54.

26. Mehta $S$, Meade $M$, Burry $L$, et al. Variation in diurnal sedation in mechanically ventilated patients who are managed with a sedation protocol alone or a sedation protocol and daily interruption. Crit Care. 2016;20:233

27. Dessap AM, Roche-Campo F, Launay JM, et al. Delirium and circadian rhythm of melatonin during weaning from mechanical ventilation: an ancillary study of a weaning trial. Chest. 2015;148:1231-41.

28. Papaioannou V, Mebazaa A, Plaud B, Legrand M. 'Chronomics' in ICU: circadian aspects of immune response and therapeutic perspectives in the critically ill. Intensive Care Med Exp. 2014;2:18.

29. Perras B, Meier M, Dodt C. Light and darkness fail to regulate melatonin release in critically ill humans. Intensive Care Med. 2007;33:1954-8.

30. McClave SA, Taylor BE, Martindale RG, et al. Guidelines for the provision and assessment of nutrition support therapy in the adult critically ill patient: society of critical care medicine (SCCM) and American Society for Parenteral and Enteral Nutrition (A.S.P.E.N.). J Parenter Enter Nutr. 2016:40:159-211.

31. Sveinsson IS. Postoperative psychosis after heart surgery. J Thorac Cardiovasc Surg. 1975;70:717-26.

32. Harrell RG, Othmer E. Postcardiotomy confusion and sleep loss. J Clin Psychiatry. 1987;48:445-6

33. Trompeo AC, Vidi Y, Locane MD, et al. Sleep disturbances in the critically ill patients: role of delirium and sedative agents. Minerva Anestesiol. 2011;77:604-12.

34. Lim AS, Yu L, Costa MD, Leurgans SE, Buchman AS, Bennett DA, Saper CB. Increased fragmentation of rest-activity patterns is associated with a characteristic pattern of cognitive impairment in older individuals. Sleep. 2012;35:633-40.

35. Altman MT, Knauert MP, Pisani MA. Sleep disturbances after hospitalization and critical illness: a systematic review. Ann Am Thorac Soc. 2017;14:1457-68.

36. Davydow DS, Gifford JM, Desai SV, Bienvenu OJ, Needham DM. Depression in general intensive care unit survivors: a systematic review. Intensive Care Med. 2009:35:796-809.

37. Drouot $X$, Roche-Campo F, Thille AW, et al. A new classification for sleep analysis in critically ill patients. Sleep Med. 2012;13:7-14.

38. Lim AS, Yu L, Kowgier M, Buchman AS, Bennett DA. Modification of the relationship of the apolipoprotein $E \varepsilon 4$ allele to the risk of Alzheimer disease and neurofibrillary tangle density by sleep. JAMA Neurol. 2013;70:1544-51.

39. Lim AS, Chang AM, Shulman JM, et al. A common polymorphism near PER1 and the timing of human behavioral rhythms. Ann Neurol. 2012;72:324-34

40. Schwab KE, Ronish B, Needham DM, To AQ, Martin JL, Kamdar BB. Actigraphy to evaluate sleep in the intensive care unit. A systematic review. Ann Am Thorac Soc. 2018;15:1075-82.
41. Matthews EE. Sleep disturbances and fatigue in critically ill patients. AACN Adv Crit Care. 2011;22:204-24.

42. Taguchi T, Yano M, Kido Y. Influence of bright light therapy on postoperative patients: a pilot study. Intensive Crit Care Nurs. 2007:23:289-97.

43. Van Rompaey B, Elseviers MM, Schuurmans MJ, Shortridge-Baggett LM, Truijen S, Bossaert L. Risk factors for delirium in intensive care patients: a prospective cohort study. Crit Care. 2009;13:R77.

44. Vinzio S, Ruellan A, Perrin AE, Schlienger JL, Goichot B. Actigraphic assessment of the circadian rest-activity rhythm in elderly patients hospitalized in an acute care unit. Psychiatry Clin Neurosci. 2003;57:53-8.

45. Demoule A, Carreira S, Lavault S, et al. Impact of earplugs and eye mask on sleep in critically ill patients: a prospective randomized study. Crit Care. 2017;21:284.

46. Bourne RS, Mills GH, Minelli C. Melatonin therapy to improve nocturnal sleep in critically ill patients: encouraging results from a small randomised controlled trial. Crit Care. 2008;12:R52.

47. Fanfulla F, Delmastro M, Berardinelli A, Lupo ND, Nava S. Effects of different ventilator settings on sleep and inspiratory effort in patients with neuromuscular disease. Am J Respir Crit Care Med. 2005;172:619-24.

48. Cabello B, Thille AW, Drouot X, et al. Sleep quality in mechanically ventilated patients: comparison of three ventilatory modes. Crit Care Med. 2008;36:1749-55.

49. Parthasarathy S, Tobin MJ. Effect of ventilator mode on sleep quality in critically ill patients. Am J Respir Crit Care Med. 2002;166:1423-9.

50. Roche-Campo F, Thille AW, Drouot X, et al. Comparison of sleep quality with mechanical versus spontaneous ventilation during weaning of critically ill tracheostomized patients. Crit Care Med. 2013;41:1637-44.

\section{Ready to submit your research? Choose BMC and benefit from:}

- fast, convenient online submission

- thorough peer review by experienced researchers in your field

- rapid publication on acceptance

- support for research data, including large and complex data types

- gold Open Access which fosters wider collaboration and increased citations

- maximum visibility for your research: over $100 \mathrm{M}$ website views per year

At BMC, research is always in progress.

Learn more biomedcentral.com/submissions 